\title{
CRISPR-DT: designing gRNAs for the CRISPR-Cpf1 system with improved target efficiency and specificity
}

\author{
Houxiang Zhu ${ }^{1}$ and Chun Liang ${ }^{1,2^{*}}$ \\ ${ }^{1}$ Department of Biology, Miami University, Oxford, OH 45056, USA \\ ${ }^{2}$ Department of Computer Science and Software Engineering, Miami University, Oxford, OH 45056, USA \\ *Correspondence: liangc@miamioh.edu
}

HZ: zhuh4@miamioh.edu

CL: liangc@miamioh.edu 


\begin{abstract}
The CRISPR-Cpf1 system has been successfully applied in genome editing. However, target efficiency of the CRISPR-Cpf1 system varies among different gRNA sequences. We reanalyzed the published CRISPR-Cpf1 gRNAs data and found many sequence and structural features related to their target efficiency. Using machine learning technology, a SVM model was created to predict target efficiency for any given gRNAs. We have developed the first web service application, CRISPR-DT (CRISPR DNA Targeting), to help users design optimal gRNAs for the CRISPR-Cpf1 system by considering both target efficiency and specificity. CRISPR-DT is available at http://bioinfolab.miamioh.edu/CRISPR-DT.
\end{abstract}

Keywords: CRISPR-Cpf1, Genome editing, Target efficiency, Target specificity 


\section{Background}

The CRISPR-Cas9 (Clustered Regularly Interspaced Short Palindromic Repeats - CRISPR associated protein 9) system is a prokaryotic immune system, which has been widely applied in eukaryotic genome editing. The basic mechanism of CRISPR-Cas9 editing is that a single guide RNA (gRNA) guides Cas9 to a DNA target sequence, then Cas9 cleaves double strands at the same position. The DNA double-strand breaks can be repaired either by non-homologous end joining (NHEJ), leading to a gene knockout, or by homology directed repair (HDR), generating a gene knockin. In 2013, the CRISPR-Cas9 system was first demonstrated as a tool for eukaryotic genome editing [1,2]. Afterwards, many studies have successfully applied CRISPR-Cas9 to engineer genomes of different organisms [3-11]. In addition, researchers found that CRISPR-Cas9 can even be used to correct genetic diseases $[12,13]$ and develop cancer models $[6$, 14-16]. But the CRISPR-Cas9 system still has some shortcomings, such as high off-target effect [17, 18]. The CRISPR-Cpf1 system was first characterized in 2015, and it is a class 2 CRISPR-Cas system with a single Cas protein to mediate cleavage like CRISPR-Cas9 [19]. Researchers found that CRISPR-Cpf1 has some characteristics distinct from those of CRISPR-Cas9 [19]. First, Cpf1 has RNase III activity for processing the precursor CRISPR RNA (pre-crRNA) into mature crRNAs [20, 21]. Second, Cpf1 needs only one crRNA for cleavage, whereas Cas9 requires both the crRNA and trans-activating crRNA (tracrRNA) [19]. Third, Cpf1 recognizes a T-rich protospacer adjacent motif (PAM), which is at the 5' end of the protospacer sequence [19]. Fourth, Cpf1 cleaves double-strand DNA via a staggered cut that generates two sticky ends, and the cleavage sites are further away from the PAM [19]. Recently, the CRISPR-Cpf1 system has been successfully applied for genome editing in various organisms. Studies showed that CRISPR-Cpf1 is highly specific in mammals [22-25]. Compared with Cas9, Cpf1 induced less off targets in human cells [23]. Cpf1 also has high target specificity in plant cells [26-29]. In addition, CRISPR-Cpf1 has been used to genetically engineer some important bacteria, such as Corynebacterium glutamicum [30] and Cyanobacteria [31], whose genomes cannot be edited by the CRISPR-Cas9 system probably due to Cas9 toxicity in these bacteria [30, 32]. Moreover, researchers demonstrated that 
CRISPR-Cpf1 is highly suitable for multiplex gene editing [21,33]. CRISPR-Cas9 can also be used in multiplex gene editing, but it requires larger constructs or delivery of several plasmids [34-37], which might cause problems in multiplex gene editing [21]. Additionally, the CRISPR-Cpf1 system has been applied in correction of genetic mutations [38, 39], which shows great therapeutic potential. Furthermore, the inactive Cpfl can be used for gene repression [26, 40]. All these studies demonstrate that the CRISPR-Cpf1 system has many advantages over CRISPR-Cas9, and it is a powerful tool for genome editing.

Target efficiency and specificity are the two most important aspects for genome editing. However, target efficiency of the CRISPR-Cpf1 system varies among different gRNA sequences [41], just like the CRISPR-Cas9 system [7, 8, 42-46]. Kim and his colleagues attempted to find out gRNA features that are related to target efficiency of CRISPR-Cpf1 [41]. They measured activities of 1251 gRNA sequences for the CRISPR-Cpf1 system and developed an algorithm to predict target efficiency [41]. In our study, we reanalyzed this public data by comparing the most efficient gRNAs (top 10\% in activity ranking) with the least efficient gRNAs (bottom 10\%). The most distinct gRNA features related to target efficiency can be identified by excluding gRNAs with modest efficiency, which has been demonstrated as a great strategy in previous studies $[47,48]$. We found many novel gRNA sequence and structural features that are highly beneficial to target efficiency of CRISPR-Cpf1. Using machine learning technology, we created a support vector machine (SVM) model to predict target efficiency by combining all the important features selected by Random Forest. Previous research showed that the SVM had robust performance in predicting target efficiency for the CRISPR-Cas9 system [48]. Random Forest can rapidly screen optimal features [49], which has been applied in various biological studies [50-52]. We have developed the first web service application, CRISPR-DT, to help users design gRNAs for the CRISPR-Cpf1 system by considering both target efficiency and specificity. 


\section{Results}

\section{Sequence features of efficient and inefficient gRNAs}

\section{Position-specific nucleotide composition}

Fig. 1 shows that within the 23-nt gRNA sequence, uracil (U) is extremely disfavored at the first position of efficient gRNAs, which is immediately adjacent to the PAM sequence $(P=2.44 \mathrm{E}-14)$. But guanine (G) and cytosine (C) are strongly favored at the first position $(P=6.96 \mathrm{E}-06$ and $P=1.14 \mathrm{E}-04)$. In the last position, efficient gRNAs prefer cytosine $(P=5.42 \mathrm{E}-06)$, but not guanine $(P=3.16 \mathrm{E}-04)$. Guanine is especially disfavored at the end of efficient gRNAs (position 18 - 23). Overall, within the 23-nt RNA sequence, efficient gRNAs favor adenine (A) but disfavor guanine except at the first position, probably due to the first position is adjacent to the PAM sequence. Position-specific dinucleotides were also analyzed by comparing efficient and inefficient gRNAs (Additional file 1: Table S1). For the first two positions of efficient gRNAs, CC and GG are favored with enrichment ratios of $4.57(P=1.12 \mathrm{E}-05)$ and $3.00(P=4.94 \mathrm{E}-03)$, whereas UC, UU, and UG are disfavored with enrichment ratios of $0.05(P=$ 1.51E-05), $0.00(P=7.08 \mathrm{E}-05)$, and $0.08(P=9.91 \mathrm{E}-04)$, respectively.

\section{Position-nonspecific nucleotide composition}

Table 1 shows that the average counts of guanine for efficient and inefficient gRNAs are 5.66 and 7.53, respectively, within the 23-nt gRNA sequence $(P=2.59 \mathrm{E}-08)$, which indicates efficient gRNAs disfavor guanine. But compared with inefficient gRNAs, efficient gRNAs have a preference for adenine (5.50 versus $4.30, P=1.57 \mathrm{E}-06)$ and cytosine (7.04 versus $6.01, P=1.24 \mathrm{E}-03)$. The most significant positionnonspecific dinucleotide count is the GG count (1.30E-06), which greatly decreased in efficient gRNAs with an enrichment ratio of 0.59 . This finding is consistent with previous study of CRISPR-Cas9 [48]. The position-nonspecific trinucleotide and tetranucleotide counts were also calculated (Additional file 1: Table S2). UGG is the most significant trinucleotide for efficient and inefficient gRNAs with average counts of 0.32 and 0.75 , respectively $(P=1.83 \mathrm{E}-05)$. The average count of GGG is significantly decreased in efficient gRNAs with an enrichment ratio of $0.57(P=8.09 \mathrm{E}-03)$, which is consistent with 
previous CRISPR-Cas9 study [48]. CCCA is the most significant tetranucleotide for efficient and inefficient gRNAs with average counts of 0.17 and 0.02 , respectively $(P=3.84 \mathrm{E}-04)$. Previous work has demonstrated that GGGG within the 23-nt gRNA sequence can cause poor CRISPR-Cas9 activity because GGGG badly affects DNA oligo synthesis, and can form a secondary structure called guanine tetrad in the gRNA sequence, which makes gRNAs difficult to bind to target sequences [48]. Consistently, for the CRISPR-Cpf1 system, we found there are more GGGG in inefficient gRNAs than that in efficient gRNAs with an enrichment ratio of $0.21(P=4.48 \mathrm{E}-04)$. Research also showed that repetitive nucleobases (at least four A, four $\mathrm{C}$, four $\mathrm{G}$, or four $\mathrm{U}$ ) are related to poor CRISPR-Cas9 activity [48]. Similarly, in our study we found more inefficient gRNAs contains repetitive nucleobases than efficient ones $(0.30$ versus $0.17, P=1.65 \mathrm{E}-02)$. In addition, we examined UUU in the gRNA seed region, which are the 6 nucleotides in the 5' PAM-proximal region [41], and found that more inefficient gRNAs contains UUU in the seed region than efficient ones with an enrichment ratio of $0.29(P=9.07 \mathrm{E}-$ 02), which is consistent with previous CRISPR-Cas9 research [48].

Table 1 Significant position-nonspecific mononucleotide and dinucleotide counts within the 23-nt gRNA sequences

\begin{tabular}{lllll}
\hline Mono- and Dinucleotides & Efficient gRNAs & Inefficient gRNAs & Enrichment Ratio $^{\mathrm{a}}$ & $P$-value \\
\hline Count_G & 5.66 & 7.53 & 0.75 & $2.59 \mathrm{E}-08$ \\
Count_A & 5.50 & 4.30 & 1.28 & $1.57 \mathrm{E}-06$ \\
Count_C & 7.04 & 6.01 & 1.17 & $1.24 \mathrm{E}-03$ \\
Count_GG & 1.19 & 2.03 & 0.59 & $1.30 \mathrm{E}-06$ \\
Count_CG & 1.10 & 1.74 & 0.64 & $1.72 \mathrm{E}-05$ \\
Count_AC & 1.14 & 1.48 & 1.44 & $9.63 \mathrm{E}-05$ \\
Count_UA & 1.01 & 0.64 & 1.58 & $1.08 \mathrm{E}-03$ \\
Count_AU & 0.79 & 0.49 & 1.62 & $1.23 \mathrm{E}-03$ \\
Count_CU & 1.67 & 1.23 & 1.36 & $1.96 \mathrm{E}-03$ \\
Count_CC & 1.68 & 1.22 & 1.37 & $3.88 \mathrm{E}-03$ \\
Count_GC & 0.87 & 0.68 & 0.68 & $5.00 \mathrm{E}-03$ \\
Count_AA & 0.91 & 1.92 & 0.78 & $5.49 \mathrm{E}-03$ \\
Count_GU & 1.50 & 1.66 & 0.78 & $8.72 \mathrm{E}-03$ \\
Count_UG & 1.30 & 1.35 & 1.25 & $1.28 \mathrm{E}-02$ \\
Count_CA & 1.70 & & $1.73 \mathrm{E}-02$ \\
\hline
\end{tabular}

${ }^{\mathrm{a}}$ The enrichment ratio was calculated through dividing the average nucleotide counts of efficient gRNAs by that of inefficient gRNAs.

${ }^{\mathrm{b}}$ The $P$-value was determined by Welch's t-test $(P<0.05)$. 


\section{GC content}

Researchers have demonstrated that the 23-nt gRNA sequence of CRISPR-Cpf1 should be divided into three regions: seed (6 nucleotides in the 5' PAM-proximal region), trunk (12 nucleotides in the middle region), and promiscuous (5 nucleotides in the 3' PAM-distal region) [41], which is illustrated in Additional file 1: Figure S1. The tolerant ability of mismatches gradually increases from the seed region to the promiscuous region. Here we separately compared GC content of the entire 23-nt gRNA sequence, seed region, trunk region, and promiscuous region between efficient and inefficient gRNAs. For the entire 23-nt gRNA sequence, inefficient gRNAs have higher GC content than efficient ones ( 0.59 versus $0.55, P=3.54 \mathrm{E}-03)$. In addition, the GC content of inefficient gRNAs is higher than that of efficient ones in both trunk and especially promiscuous regions $(0.58$ versus $0.54, P=7.41 \mathrm{E}-03$ and 0.64 versus $0.52, P=8.16 \mathrm{E}-06)$, but there is no big difference of GC content in the seed region. Since gRNAs with balanced GC content have higher target efficiency for the CRISPR-Cas9 system [8, 42, 53], in our study we consider GC content of 0.30 - 0.70 as normal, but greater than 0.70 or less than 0.30 as abnormal. Results showed that more efficient gRNAs contain normal GC content than inefficient ones $(0.98$ versus $0.89, P=1.95 \mathrm{E}-03)$.

\section{Structural features of efficient and inefficient gRNAs}

\section{Minimum free energy}

The secondary structure stability of the 23-nt gRNA sequence was determined by minimum free energy (MFE). Compared with efficient gRNAs, more inefficient gRNAs have lower MFE but fewer ones have higher MFE (Fig. 2). In addition, the average MFE of inefficient gRNAs is significantly lower than that of efficient ones $(-3.89$ versus $-1.81, P=1.10 \mathrm{E}-11)$, which means the secondary structure of inefficient gRNAs is more stable than that of efficient ones. Our finding indicates that nucleotide accessibility of the 23-nt gRNA sequence is strongly related to target efficiency, which is consistent with previous CRISPRCas9 study [48]. 


\section{Melting temperature}

The melting temperature was calculated based on the DNA version of the gRNA sequence [54]. We separately compared melting temperatures of the entire 23-nt gRNA sequence, seed region, trunk region, and promiscuous region between efficient and inefficient gRNAs, which is a similar strategy as previous research [54]. For the entire 23-nt gRNA sequence, inefficient RNAs have a significantly higher average melting temperature than efficient ones (63.85 versus $61.34, P=1.29 \mathrm{E}-06)$. Additionally, the average melting temperatures of inefficient gRNAs are higher than those of efficient ones in both trunk (38.03 versus $35.96, P=5.37 \mathrm{E}-03)$ and promiscuous $(-24.14$ versus $-31.84, P=1.58 \mathrm{E}-07)$ regions, but not in the seed region.

\section{A SVM model to predict target efficiency}

The top $10 \%$ and bottom $10 \%$ (250 total) gRNAs in activity ranking of the 1251 gRNAs were used to train and test our support vector machine (SVM) models. Since the full set of sequence and structural features was overdetermined, feature selection was performed before training SVM models. We used ten-fold cross validation to evaluate the SVM models and the receiver operating characteristic (ROC) curves are shown in Fig. 3. The average area under the curve (AUC) is 0.87 , which means the SVM models have high performance in distinguishing efficient and inefficient gRNAs. We built a final SVM model by using all 250 gRNAs with the most important features. To further validate the SVM model, we compared our model with the previously published model [41], which was based on 104 independent gRNAs data (Fig. 4a). Our model showed to have a better performance than Kim et al.'s model (AUC = 0.76 versus 0.67 ) in predicting target efficiency using 104 independent gRNAs. Since research has demonstrated that TTTA is the preferred PAM sequence for the CRISPR-Cpf1 system relative to other PAM sequences [41], it's very important to evaluate our model by only using gRNAs with the TTTA PAM. Fig. $4 b$ shows our model has a better performance than Kim et al.'s model (AUC $=0.79$ versus 0.44) in predicting target efficiency using 35 independent gRNAs with the TTTA PAM. Additionally, we evaluated our model based on gRNAs with TTTC and TTTG PAMs, separately (Additional file 1: Figure S2). Our model performs better at gRNAs with the TTTC PAM than Kim et al.'s model (AUC = 
0.78 versus 0.71 ) and both models show similar results on gRNAs with the TTTG PAM (AUC $=0.71$

versus 0.72 ). In summary, our SVM model has robust performance at predicting target efficiency.

\section{A web service application for improving target efficiency and specificity}

Though the CRISPR-Cpf1 system is highly specific in human and plant cells, we still can use bioinformatics methods to improve target specificity at the greatest extent. By considering both target efficiency and specificity, we have developed a web service application, CRISPR-DT, to help users design optimal gRNAs for the CRISPR-Cpf1 system. In the setting page (Additional file 1: Figure S3), first, users input a DNA sequence they want to target in FASTA format. Second, users select a reference genome. Third, users set on- and off-target PAMs, respectively. Fourth, users choose an off-target setting. Then they can click "Find targets!" to run the program. In the result pages, Fig. 5a shows all the target candidates and relevant information, including the number of target sites within the entire genome, the number of exon targets within the entire genome, and the efficiency score of each target candidate. Users can rank target candidates by clicking column headers and choose optimal ones by considering both offtarget effect and target efficiency. The number of target sites and the number of exon targets can be clicked to show the detailed information for each target site (Fig. 5b), including alignment information and the JBrowse [55] link, which can be clicked to display the target site in the background of genomic and transcript features (Fig. 5c).

\section{Discussion}

The CRISPR-Cas9 system has been widely applied in genome editing. More recently, the CRISPR-Cpf1 system was identified as a new powerful tool for genome engineering [19]. The CRISPR-Cpf1 system is a class 2 CRISPR-Cas system like CRISPR-Cas9, but it has some distinct characteristics. For example, Cpf1 recognizes a T-rich PAM and cleaves double-strand DNA via a staggered cut, resulting in two sticky ends [19]. Compared with CRISPR-Cas9, the CRISPR-Cpf1 system has some advantages, such as higher target specificity in human cells [23] and better performance in multiplex gene editing [21]. 
Target efficiency and specificity are the two most important aspects for genome editing. However, target efficiency for the CRISPR-Cpfl system varies among different gRNAs [41]. gRNA characteristics related to target efficiency have not been well studied. Here, we reanalyzed the published CRISPR-Cpf1 gRNAs data [41] and found that many sequence and structural features of gRNAs (e.g., the positionspecific nucleotide composition, position-nonspecific nucleotide composition, GC content, minimum free energy, and melting temperature) are correlated with target efficiency. As a machine learning technology, a SVM model was created based on the published gRNAs data to predict target efficiency for any given gRNAs. We have developed the first web service application, CRISPR-DT, to help users design optimal gRNAs for the CRISPR-Cpf1 system by considering both target efficiency and specificity. The target efficiency score is available for mammals because the SVM model was built based on mammalian data [41]. We have updated our previously published three CRISPR-Cas systems (Cas9, Cas9n, and RFN) by incorporating Doench et al.'s model to predict target efficiency for mammals [54, 56], which are also available in CRISPR-DT. CRISPR-DT will empower researchers in genome editing.

\section{Methods}

\section{Data retrieval and usage}

The published 1251 gRNAs data was downloaded from the journal's website (https://www.nature.com/articles/nmeth.4104) [41]. The top 10\% (125 efficient) and bottom 10\% (125 inefficient) gRNAs in activity ranking of the 1251 gRNAs were analyzed to explore features related to target efficiency and were also used to train the SVM model. In addition, the 104 independent gRNAs data for validating the SVM model was downloaded from the journal's website [41]. The reference genomes and corresponding gff3 annotation files for predicting target specificity were downloaded from Ensembl (https://useast.ensembl.org) and Ensembl Plants (https://plants.ensembl.org) with release 81 for animals and release 27 for plants. 


\section{Computational tools and statistical analysis}

The minimum free energy of each 23-nt gRNA sequence was calculated by RNAfold with default parameters [57]. The Melting temperatures of the entire 23-nt gRNA sequence, seed region, trunk region, and promiscuous region were calculated using the Biopython Tm_NN function with thermodynamic values from the DNA_NN2 table [58-60]. Welch's t-test was used to perform statistical significance analysis $(P$-value $<0.05)$ by the R package.

\section{Target efficiency predictive model}

The top $10 \%$ (125) gRNAs in activity ranking of the 1251 gRNAs were labeled by "efficient", while the bottom 10\% (125) gRNAs were labeled by "inefficient". We calculated 796 features for the 250 gRNAs, including the position-specific nucleotide composition $\left(23 \times 4+22 \times 4^{2}\right)$, position-nonspecific nucleotide composition $\left(4+4^{2}+4^{3}+4^{4}+1+1\right)$, GC content $(4+1)$, minimum free energy $(1)$, and melting temperature (4). Since the full set of gRNA features was overdetermined, feature selection was preformed carefully using Random Forest before training SVM models. We used ten-fold cross validation to evaluate the SVM model performance. Specifically, the 250 efficient and inefficient gRNAs were randomly divided into ten folds; each fold was used once as the test data and correspondingly, the remaining nine folds were used as the training data. For each time, first, we used Random Forest to select important features (48 features were selected according to the mean decrease impurity) based on the training data; second, optimal parameters of the SVM model were selected following the protocol recommended by LIBSVM [61]; third, we built the SVM model by LIBSVM, using a radial basis function (RBF) as kernel transformation, based on the training data with the important features and optimal parameters; fourth, the SVM model was evaluated by the test data. After repeating the above procedure ten times, we got the average performance of SVM models. The ROCR package of R was used to draw the ROC curve and calculate the AUC value. Last, we built the final SVM model by using all the 250 gRNAs data with the 48 most important features (Additional file 1: Table S3) and optimal parameters. To further validate the final SVM model, we compared our model with previously published model by using 104 independent gRNAs data, in which the top $20 \%$ gRNAs in activity ranking were 
defined as efficient ones and the remaining $80 \%$ gRNAs were regarded as inefficient ones [41]. In addition, the 104 gRNAs were divided into three subsets based on different PAM sequences (TTTA, TTTC, and TTTG) to further evaluate the SVM model. In each subset, the top $20 \%$ gRNAs in activity ranking were defined as efficient ones and the remaining $80 \%$ gRNAs were regarded as inefficient ones $[41]$.

\section{Bioinformatics pipeline for improving target specificity}

The pipeline is shown in Additional file 1: Figure S4. First, users input a DNA sequence. Second, a Perl script was used to search all target candidates from the input DNA sequence based on the on-target PAM sequence users set. Third, each target candidate is mapped to the reference genome by Bowtie2 [62] to find all the possible target sites in the genome according to the setting of maximum number of mismatches and gaps that off targets can tolerate. Fourth, samtools [63] was used to separate alignment results to the forward strand and reverse strand. Fifth, several Perl scripts were used to filter results based on users off-target settings. After converting file format, we use PHP and JavaScript to display the results in DataTables and JBrowse [55]. The same strategy was used in our previous work [56, 64]. 


\section{Figures}

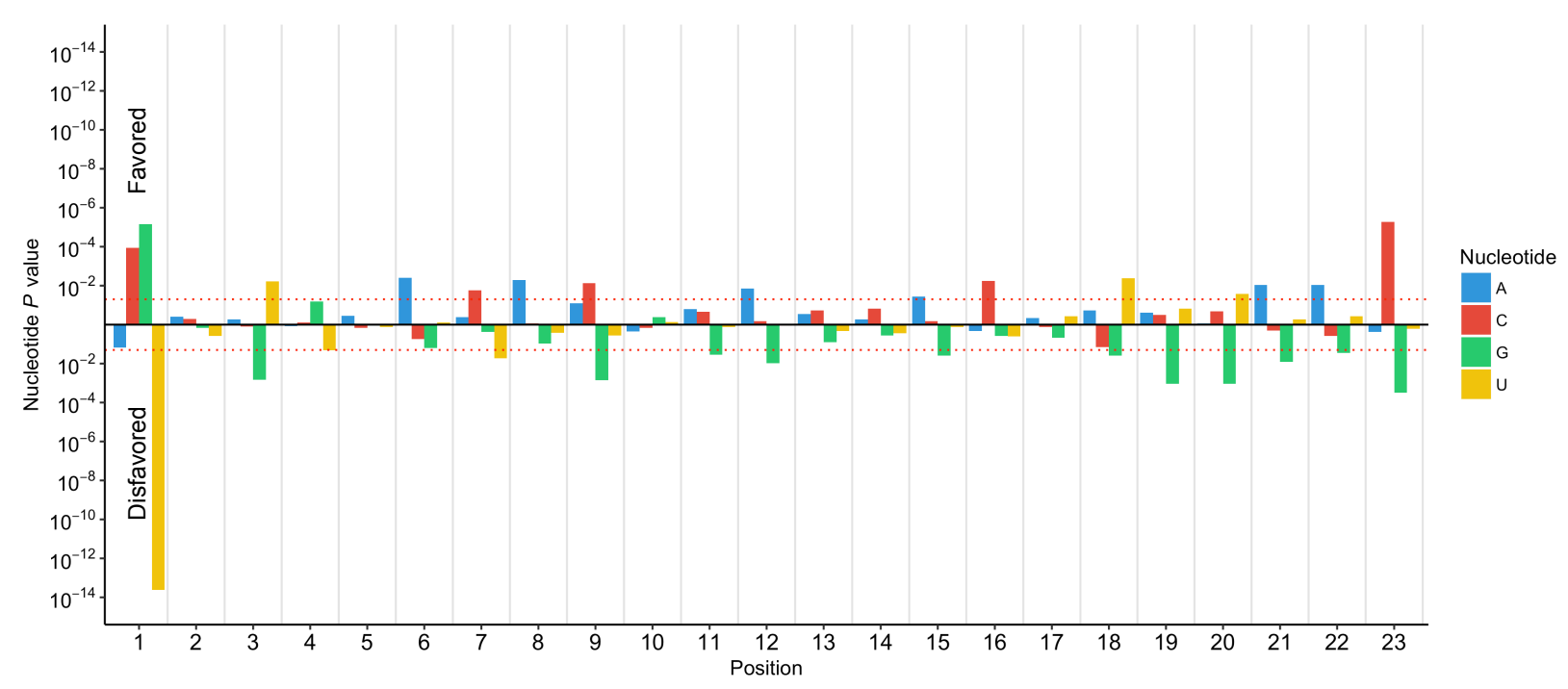

Fig. $1 P$-values of single nucleotides at each position of the 23 -nt gRNA sequence. The $\mathrm{Y}$ axis indicates whether a single nucleotide is favored or disfavored by efficient gRNAs. The red lines are used as the cutoff for significance $(P=0.05)$. 


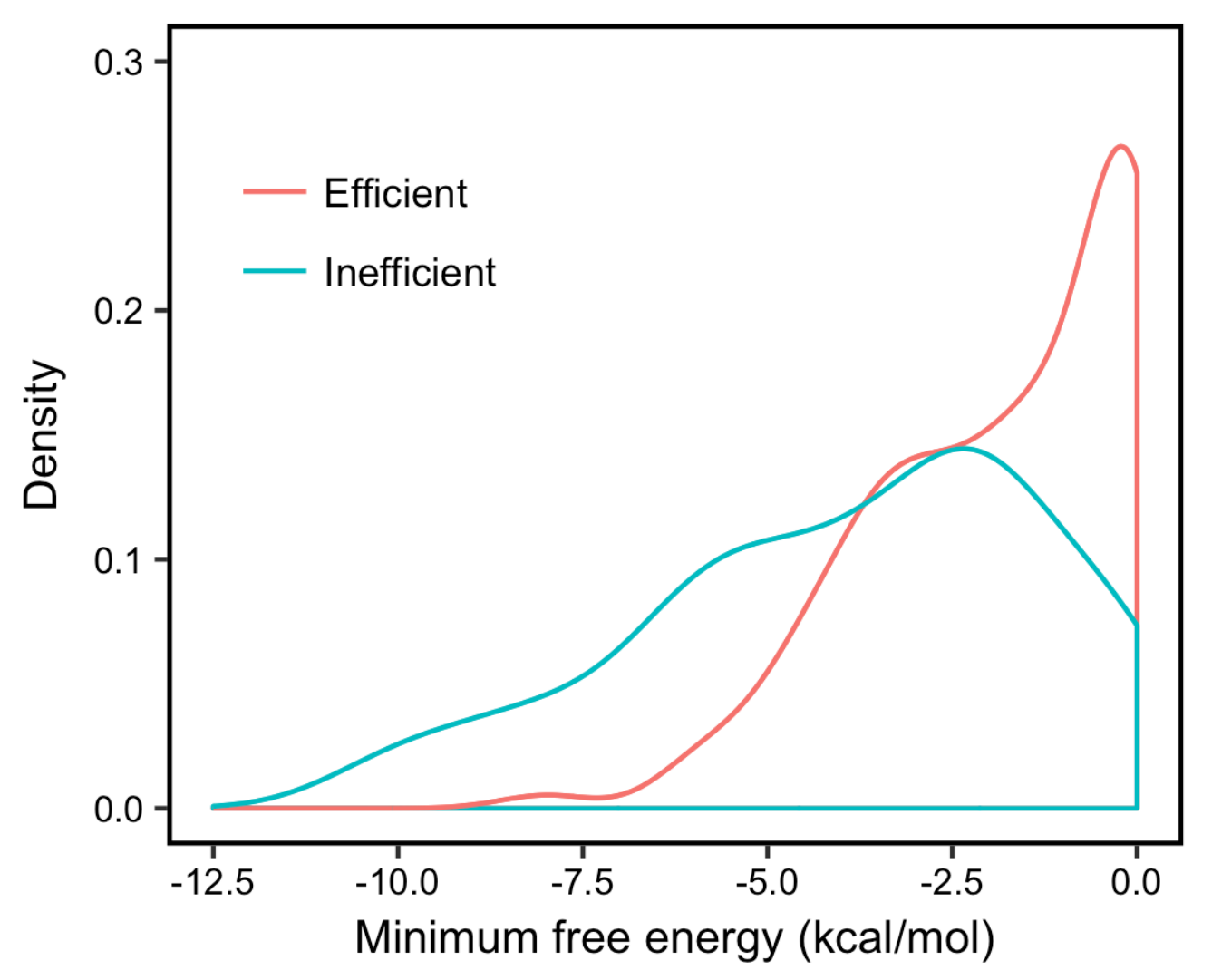

Fig. 2 The distribution of efficient and inefficient gRNAs for minimum free energy. 


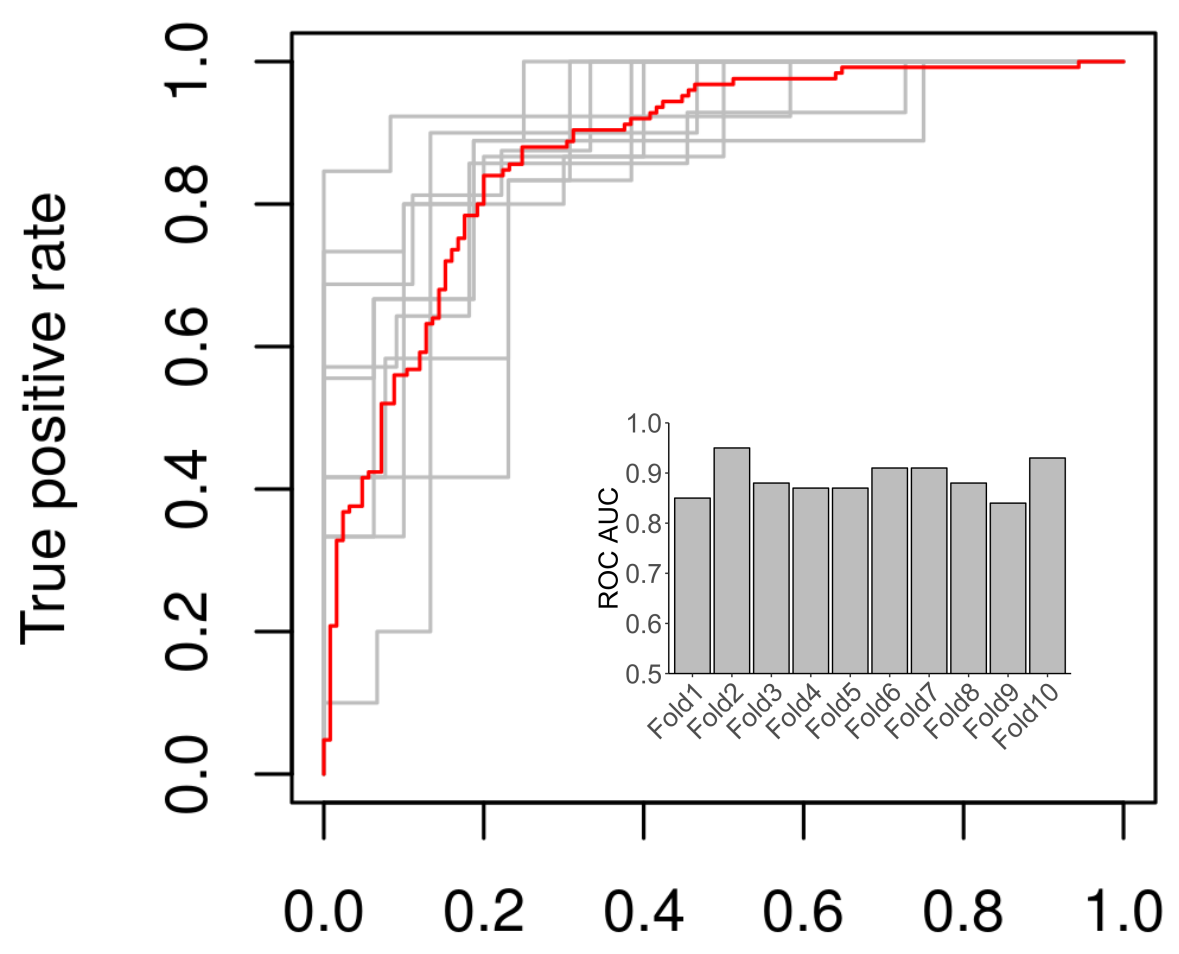

False positive rate

Fig. 3 Performance evaluation of SVM models. Ten-fold cross validation was used to evaluate the SVM models. Each gray line indicates the ROC curve for each fold. The red line is the average ROC curve. The bar graph indicates the AUC for each fold. 
a

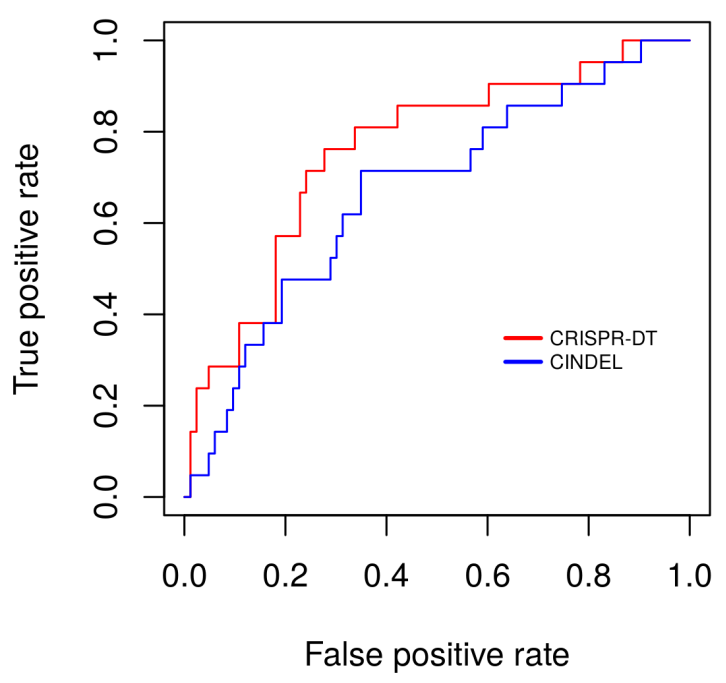

b

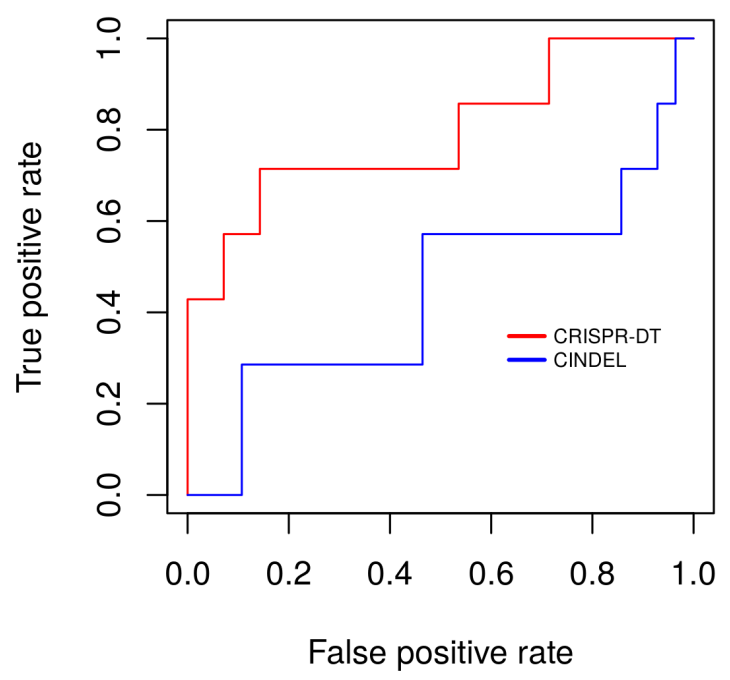

Fig. 4 Validation of our final SVM model using independent experimental data. a ROC curves comparing the performance of our model (CRISPR-DT) and Kim et al.'s model (CINDEL) in predicting target efficiency using 104 independent gRNAs. b ROC curves comparing the performance of our model (CRISPR-DT) and Kim et al.'s model (CINDEL) in predicting target efficiency using independent gRNAs with the TTTA PAM. 
bioRxiv preprint doi: https://doi.org/10.1101/269910; this version posted February 22, 2018. The copyright holder for this preprint (which was not certified by peer review) is the author/funder, who has granted bioRxiv a license to display the preprint in perpetuity. It is made available under aCC-BY-NC-ND 4.0 International license.



Fig. 5 The result web interfaces of CRISPR-DT. a The detailed information of all the target candidates in the user input DNA sequence. $\mathbf{b}$ The detailed information of target sites in the reference genome for each target candidate. c Visualization of on- and off-target sites with genomic and transcript annotations in JBrowse. 


\section{Declarations}

\section{Ethics approval and consent to participate}

Not applicable

\section{Consent for publication}

Not applicable

\section{Availability of data and material}

CRISPR-DT is freely accessible via the bioinformatics laboratory website of Miami University [65]. The source code is available from the authors on request.

\section{Competing interests}

The authors declare that they have no competing interests.

\section{Funding}

This project is funded by Office for the Advancement of Research and Scholarship (OARS) and Biology Department, Miami University, Oxford, OH, USA.

\section{Authors' contributions}

CL managed and coordinated the whole project. HZ and CL participated in CRISPR-DT design. HZ implemented statistical analysis, SVM modeling, and the backend pipeline in Perl. HZ and CL participated in web interfaces implementation. HZ and CL prepared the manuscript. All authors have read and approved the final manuscript.

\section{Acknowledgements}

This project is funded by Office for the Advancement of Research and Scholarship (OARS) and Biology Department, Miami University, Oxford, OH, USA.

\section{Additional file}

Additional file 1: Supplementary Figures and Tables. (PDF $572 \mathrm{~kb}$ ) 


\section{References}

1. Cong L, Ran FA, Cox D, Lin S, Barretto R, Habib N, et al. Multiplex Genome Engineering Using CRISPR/Cas Systems. Science. 2013;339:819-23.

2. Mali P, Yang L, Esvelt KM, Aach J, Guell M, DiCarlo JE, et al. RNA-Guided Human Genome Engineering via Cas9. Science. 2013;339:823-6.

3. Friedland AE, Church GM, Calarco JA, Esvelt KM, Colaiácovo MP, Tzur YB. Heritable genome editing in $C$. elegans via a CRISPR-Cas9 system. Nature Methods. 2013;10:741.

4. Bassett AR, Tibbit C, Ponting CP, Liu J-L. Highly Efficient Targeted Mutagenesis of Drosophila with the CRISPR/Cas9 System. Cell Reports. 2013;4:220-8.

5. Ma Y, Zhang X, Shen B, Lu Y, Chen W, Ma J, et al. Generating rats with conditional alleles using CRISPR/Cas9. Cell Res. 2014;24:122-5.

6. Platt RJ, Chen S, Zhou Y, Yim MJ, Swiech L, Kempton HR, et al. CRISPR-Cas9 Knockin Mice for Genome Editing and Cancer Modeling. Cell. 2014;159:440-55.

7. Shalem O, Sanjana NE, Hartenian E, Shi X, Scott DA, Mikkelsen TS, et al. Genome-Scale CRISPR-Cas9

Knockout Screening in Human Cells. Science. 2014;343:84-7.

8. Wang T, Wei JJ, Sabatini DM, Lander ES. Genetic Screens in Human Cells Using the CRISPR-Cas9 System. Science. 2014;343:80-4.

9. Li J-F, Aach J, Norville JE, McCormack M, Zhang D, Bush J, et al. Multiplex and homologous recombinationmediated plant genome editing via guide RNA/Cas9. Nature biotechnology. 2013;31:688.

10. Shan Q, Wang Y, Li J, Zhang Y, Chen K, Liang Z, et al. Targeted genome modification of crop plants using a CRISPR-Cas system. Nat. Biotechnol. 2013;31:686-8.

11. Feng Z, Zhang B, Ding W, Liu X, Yang D-L, Wei P, et al. Efficient genome editing in plants using a CRISPR/Cas system. Cell Research. 2013;23:1229.

12. Wu Y, Liang D, Wang Y, Bai M, Tang W, Bao S, et al. Correction of a Genetic Disease in Mouse via Use of CRISPR-Cas9. Cell Stem Cell. 2013;13:659-62.

13. Long C, McAnally JR, Shelton JM, Mireault AA, Bassel-Duby R, Olson EN. Prevention of muscular dystrophy in mice by CRISPR/Cas9-mediated editing of germline DNA. Science. 2014;345:1184-8.

14. Matano M, Date S, Shimokawa M, Takano A, Fujii M, Ohta Y, et al. Modeling colorectal cancer using CRISPRCas9-mediated engineering of human intestinal organoids. Nat. Med. 2015;21:256-62.

15. Xue W, Chen S, Yin H, Tammela T, Papagiannakopoulos T, Joshi NS, et al. CRISPR-mediated direct mutation of cancer genes in the mouse liver. Nature. 2014;514:380.

16. Heckl D, Kowalczyk MS, Yudovich D, Belizaire R, Puram RV, McConkey ME, et al. Generation of mouse models of myeloid malignancy with combinatorial genetic lesions using CRISPR-Cas9 genome editing. Nature biotechnology. 2014;32:941.

17. Fu Y, Foden JA, Khayter C, Maeder ML, Reyon D, Joung JK, et al. High frequency off-target mutagenesis induced by CRISPR-Cas nucleases in human cells. Nature biotechnology. 2013;31:822.

18. Cradick TJ, Fine EJ, Antico CJ, Bao G. CRISPR/Cas9 systems targeting $\beta$-globin and CCR5 genes have substantial off-target activity. Nucleic Acids Res. 2013;41:9584-92.

19. Zetsche B, Gootenberg JS, Abudayyeh OO, Slaymaker IM, Makarova KS, Essletzbichler P, et al. Cpf1 Is a Single RNA-Guided Endonuclease of a Class 2 CRISPR-Cas System. Cell. 2015;163:759-71.

20. Fonfara I, Richter H, Bratovič M, Le Rhun A, Charpentier E. The CRISPR-associated DNA-cleaving enzyme Cpf1 also processes precursor CRISPR RNA. Nature. 2016;532:517-21.

21. Zetsche B, Scott DA, DeGennaro EM, Zhang F, Fedorova I, Kneppers J, et al. Multiplex gene editing by CRISPR-Cpf1 using a single crRNA array. Nature Biotechnology. 2017;35:31.

22. Kleinstiver BP, Joung JK, Lopez JM, Aryee MJ, Prew MS, Welch MM, et al. Genome-wide specificities of CRISPR-Cas Cpf1 nucleases in human cells. Nature Biotechnology. 2016;34:869.

23. Kim D, Kim J-S, Kim J, Hur JK, Been KW, Yoon S. Genome-wide analysis reveals specificities of Cpf1 endonucleases in human cells. Nature Biotechnology. 2016;34:863.

24. Kim Y, Cheong S-A, Lee JG, Lee S-W, Lee MS, Baek I-J, et al. Generation of knockout mice by Cpf1-mediated gene targeting. Nat. Biotechnol. 2016;34:808-10.

25. Hur JK, Kim K, Been KW, Baek G, Ye S, Hur JW, et al. Targeted mutagenesis in mice by electroporation of Cpf1 ribonucleoproteins. Nat. Biotechnol. 2016;34:807-8.

26. Tang X, Lowder LG, Zhang T, Malzahn AA, Zheng X, Voytas DF, et al. A CRISPR-Cpf1 system for efficient genome editing and transcriptional repression in plants. Nat Plants. 2017;3:17018. 
27. Xu R, Qin R, Li H, Li D, Li L, Wei P, et al. Generation of targeted mutant rice using a CRISPR-Cpf1 system. Plant Biotechnol J. 2017;15:713-7.

28. Hu X, Wang C, Liu Q, Fu Y, Wang K. Targeted mutagenesis in rice using CRISPR-Cpf1 system. J Genet Genomics. 2017;44:71-3.

29. Kim H, Kim S-T, Ryu J, Kang B-C, Kim J-S, Kim S-G. CRISPR/Cpf1-mediated DNA-free plant genome editing. Nature Communications. 2017;8:14406.

30. Jiang Y, Qian F, Yang J, Liu Y, Dong F, Xu C, et al. CRISPR-Cpf1 assisted genome editing of Corynebacterium glutamicum. Nat Commun. 2017;8:15179.

31. Ungerer J, Pakrasi HB. Cpf1 Is A Versatile Tool for CRISPR Genome Editing Across Diverse Species of Cyanobacteria. Scientific Reports. 2016;6:39681.

32. Wendt KE, Ungerer J, Cobb RE, Zhao H, Pakrasi HB. CRISPR/Cas9 mediated targeted mutagenesis of the fast growing cyanobacterium Synechococcus elongatus UTEX 2973. Microbial Cell Factories. 2016;15:115.

33. Wang M, Mao Y, Lu Y, Tao X, Zhu J. Multiplex Gene Editing in Rice Using the CRISPR-Cpf1 System. Molecular Plant. 2017;10:1011-3.

34. Kabadi AM, Ousterout DG, Hilton IB, Gersbach CA. Multiplex CRISPR/Cas9-based genome engineering from a single lentiviral vector. Nucleic Acids Res. 2014;42:e147-e147.

35. Nissim L, Perli SD, Fridkin A, Perez-Pinera P, Lu TK. Multiplexed and Programmable Regulation of Gene Networks with an Integrated RNA and CRISPR/Cas Toolkit in Human Cells. Molecular Cell. 2014;54:698-710. 36. Sakuma T, Nishikawa A, Kume S, Chayama K, Yamamoto T. Multiplex genome engineering in human cells using all-in-one CRISPR/Cas9 vector system. Scientific Reports. 2014;4:5400.

37. Xie K, Minkenberg B, Yang Y. Boosting CRISPR/Cas9 multiplex editing capability with the endogenous tRNAprocessing system. PNAS. 2015;112:3570-5.

38. Zhang Y, Long C, Li H, McAnally JR, Baskin KK, Shelton JM, et al. CRISPR-Cpf1 correction of muscular dystrophy mutations in human cardiomyocytes and mice. Science Advances. 2017;3:e1602814.

39. Yang M, Wei H, Wang Y, Deng J, Tang Y, Zhou L, et al. Targeted Disruption of V600E-Mutant BRAF Gene by CRISPR-Cpf1. Molecular Therapy - Nucleic Acids. 2017;8:450-8.

40. Zhang X, Wang J, Cheng Q, Zheng X, Zhao G, Wang J. Multiplex gene regulation by CRISPR-ddCpf1. Cell Discovery. 2017;3:17018.

41. Kim HK, Song M, Lee J, Menon AV, Jung S, Kang Y-M, et al. In vivo high-throughput profiling of CRISPRCpf1 activity. Nat. Methods. 2017;14:153-9.

42. Doench JG, Hartenian E, Graham DB, Tothova Z, Hegde M, Smith I, et al. Rational design of highly active sgRNAs for CRISPR-Cas9-mediated gene inactivation. Nature biotechnology. 2014;32:1262.

43. Gilbert LA, Horlbeck MA, Adamson B, Villalta JE, Chen Y, Whitehead EH, et al. Genome-Scale CRISPRMediated Control of Gene Repression and Activation. Cell. 2014;159:647-61.

44. Koike-Yusa H, Li Y, Tan E-P, Velasco-Herrera MDC, Yusa K. Genome-wide recessive genetic screening in mammalian cells with a lentiviral CRISPR-guide RNA library. Nat. Biotechnol. 2014;32:267-73.

45. Konermann S, Brigham MD, Trevino AE, Joung J, Abudayyeh OO, Barcena C, et al. Genome-scale transcriptional activation by an engineered CRISPR-Cas9 complex. Nature. 2015;517:583.

46. Zhou Y, Zhu S, Cai C, Yuan P, Li C, Huang Y, et al. High-throughput screening of a CRISPR/Cas9 library for functional genomics in human cells. Nature. 2014;509:487-91.

47. Wang X, Wang X, Varma RK, Beauchamp L, Magdaleno S, Sendera TJ. Selection of hyperfunctional siRNAs with improved potency and specificity. Nucleic Acids Res. 2009;37:e152-e152.

48. Wong N, Liu W, Wang X. WU-CRISPR: characteristics of functional guide RNAs for the CRISPR/Cas9 system. Genome Biology. 2015;16:218.

49. Saeys Y, Inza I, Larrañaga P. A review of feature selection techniques in bioinformatics. Bioinformatics. 2007;23:2507-17.

50. Ebina T, Toh H, Kuroda Y. DROP: an SVM domain linker predictor trained with optimal features selected by random forest. Bioinformatics. 2011;27:487-94.

51. Lou W, Wang X, Chen F, Chen Y, Jiang B, Zhang H. Sequence Based Prediction of DNA-Binding Proteins Based on Hybrid Feature Selection Using Random Forest and Gaussian Naïve Bayes. PLOS ONE. 2014;9:e86703. 52. Everson TM, Lyons G, Zhang H, Soto-Ramírez N, Lockett GA, Patil VK, et al. DNA methylation loci associated with atopy and high serum IgE: a genome-wide application of recursive Random Forest feature selection. Genome Medicine. 2015;7:89.

53. Gagnon JA, Valen E, Thyme SB, Huang P, Ahkmetova L, Pauli A, et al. Efficient Mutagenesis by Cas9 ProteinMediated Oligonucleotide Insertion and Large-Scale Assessment of Single-Guide RNAs. PLOS ONE.

2014;9:e98186. 
54. Doench JG, Fusi N, Sullender M, Hegde M, Vaimberg EW, Donovan KF, et al. Optimized sgRNA design to maximize activity and minimize off-target effects of CRISPR-Cas9. Nat. Biotechnol. 2016;34:184-91.

55. Skinner ME, Uzilov AV, Stein LD, Mungall CJ, Holmes IH. JBrowse: A next-generation genome browser. Genome Res. 2009;19:1630-8.

56. Zhu H, Misel L, Graham M, Robinson ML, Liang C. CT-Finder: A Web Service for CRISPR Optimal Target Prediction and Visualization. Scientific Reports. 2016;6:25516.

57. Lorenz R, Bernhart SH, Siederdissen CH zu, Tafer H, Flamm C, Stadler PF, et al. ViennaRNA Package 2.0. Algorithms for Molecular Biology : AMB. 2011;6:26.

58. Cock PJA, Antao T, Chang JT, Chapman BA, Cox CJ, Dalke A, et al. Biopython: freely available Python tools for computational molecular biology and bioinformatics. Bioinformatics. 2009;25:1422-3.

59. Le Novère N. MELTING, computing the melting temperature of nucleic acid duplex. Bioinformatics. 2001;17:1226-7.

60. Sugimoto N, Nakano S, Yoneyama M, Honda K. Improved Thermodynamic Parameters and Helix Initiation Factor to Predict Stability of DNA Duplexes. Nucleic Acids Res. 1996;24:4501-5.

61. Chang C-C, Lin C-J. LIBSVM: A library for support vector machines. ACM Transactions on Intelligent Systems and Technology (TIST). 2011;2:27.

62. Langmead B, Salzberg SL. Fast gapped-read alignment with Bowtie 2. Nature Methods. 2012;9:357.

63. Li H, Handsaker B, Wysoker A, Fennell T, Ruan J, Homer N, et al. The Sequence Alignment/Map format and SAMtools. Bioinformatics. 2009;25:2078-9.

64. Zhu H, Richmond E, Liang C. CRISPR-RT: a web application for designing CRISPR-C2c2 crRNA with improved target specificity. Bioinformatics. 2018;34:117-9.

65. CRISPR-DT. http://bioinfolab.miamioh.edu/CRISPR-DT. 